\title{
Distribution of Gell Size in Growing Cultures of Bacteria and the Applicability of the Collins-Richmond Principle
}

\author{
By A. L. KOCH \\ Departments of Biochemistry and Microbiology, College of Medicine, \\ University of Florida, Gainesville, Florida, U.S.A.
}

(Received 15 March 1966)

\section{SUMMARY}

Theoretical cell-size distributions for populations of growing cells are calculated for different models of cell growth and for different degrees of variability in size of cells at division. From these computations, it is concluded that the coefficient of variation (c.v.) is almost independent of the relationship of growth rate to cell size. It is $20 \%$ if there is no variability in the cell size at division. For a case typical for enteric rod-shaped bacteria, the variability in cell size at division is about $10 \%$ and the calculated C.v. in cell size of the population in this case increases to $22-23 \%$. Calculations based on the microscopic observations of others are in the range of $20-25 \%$. It is proposed that the c.v. of the size distribution serve as a standard in assessing the accuracy of the electronic instruments that size bacteria.

Evidently, only the higher moments of the population cell size distribution contain information bearing on the growth dependence of the organisms on their size. It is pointed out that this means that the CollinsRichmond principle must be applied only to precise and accurate data.

\section{INTRODUCTION}

In a growing culture of bacteria, at any instant of time, there is a range of sizes. The measurement of the distribution of these sizes is important because it defines the laws of growth and of cell division which apply to each individual bacterium in the culture. It is evident that in balanced growth the shape of this distribution depends on several factors. First and most important, it depends on the fact that single-celled bacteria grow by binary fission into two daughters, each capable of further division. Secondly, the distribution depends on the kinetics of the growth of a single organism (the growth rate of a bacterium might depend on the time since division, on the mass of the bacterium, or on other physiological events taking place within the bacterium). Thirdly, the distribution depends on the distribution of sizes of those bacteria in the population which are in the act of division. The question is, can we, or how can we, extract information about the second and third factors from the observed size distribution?

This problem is of current interest because of the availability of electronic instruments which measure the size of bacteria in liquid suspension. All the instruments are based on the principle developed by Coulter: a suspension of particles is pumped through a small orifice, and the change in resistance as the particles enter the orifice 
is approximately proportional to the volume of the particle; pulses proportional to the resistance changes are sorted and counted electronically. Commercially available equipment (Coulter Electronic, Inc., Hialeah, Florida, U.S.A.) automatically counts the number of particles and writes out their size distribution. Great improvements in accuracy are now possible because of the improved orifice of Kubitschek (1964). In addition, improved electronics have been introduced in the research laboratories of Kubitschek, Marr and Glaser which increase the speed, reliability, accuracy, and ease of obtaining size distributions. At the present time no one can precisely define the accuracy of these various instruments. The second purpose of the present note is to supply guide lines for their utilization.

\section{Theories yielding mass distribution}

Several years ago Koch \& Schaechter (1962) derived the distribution of cell sizes on the assumption that growth of protoplasm is exponential throughout the cell division cycle, i.e. that the rate of protoplasm synthesis is directly proportional to the amount of protoplasm in the cell. This would be the logical consequence of the assumption that ribosomes are made continuously and, once made, function with constant efficiencies. When this assumption of exponential growth was combined with a second assumption that cells divide precisely when they achieve a certain critical size, the resultant distribution was found to follow an inverse square law. This particular distribution had also been calculated by McLean \& Munson (1961). Mathematically the distribution is

and

$$
\left.\begin{array}{l}
\theta(m)=\frac{\bar{c}}{m^{2}} ; \quad\left(\frac{1}{2} \bar{c} \leqslant m \leqslant \bar{c}\right) \\
\theta(m)=0 ; \quad\left(m<\frac{1}{2} \bar{c}, m>\vec{c}\right)
\end{array}\right\}
$$

where $\bar{c}$ is the size at division and $m$ is the mass of individual cells.

When the derivation is extended to include variable sizes of cells at division, the following formula is obtained:

$$
\theta(m)=\frac{C}{m^{2}}\left[\int_{o}^{2 m} g(m) d m-\int_{o}^{m} g(m) d m\right]
$$

Here $g(m)$ is the distribution of cell sizes at the instant of cell division.

More recently Powell (1964) discussed this derivation, criticized it, but came to the conclusion that it would be valid if the distribution $g(m)$ were narrow; and, in such a case, the constant $C$ is the harmonic mean of the $g(m)$ distribution. Graphical representation of equations (1) and (2) are given in Fig. 1. In the dotted line in Fig. 1, the points were computed from equations where the $g(m)$ distribution has been assumed to be normal and to have a coefficient of variation of $10 \%$. This is a value typical of the several experimental determinations reported in the literature (Koch \& Schaechter, 1962; Schaechter, Williamson, Hood \& Koch, 1962). Also shown is the curve for $20 \%$ variation.

Probably there are many circumstances in which these distributions apply, so they are presented in different graphical forms in Figs. 2 and 3. In Fig. 2, the cumulant frequency is presented on log probability paper. It is seen that the distribution approximates the log normal distribution over the central $80 \%$ of the cells for a $\mathbf{2 0} \%$ coefficient of variation (c.v.) in the size at division, and from the $\mathbf{2 0}$ 


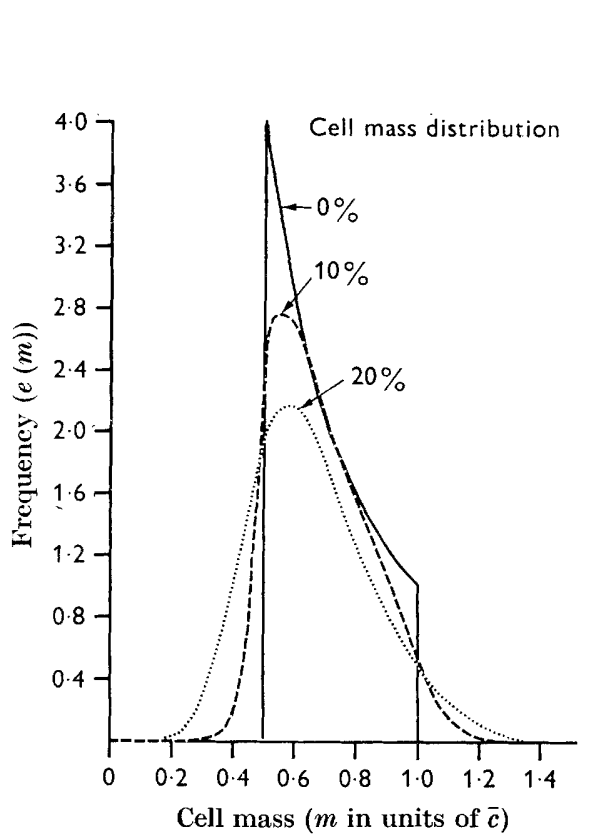

Fig. 1

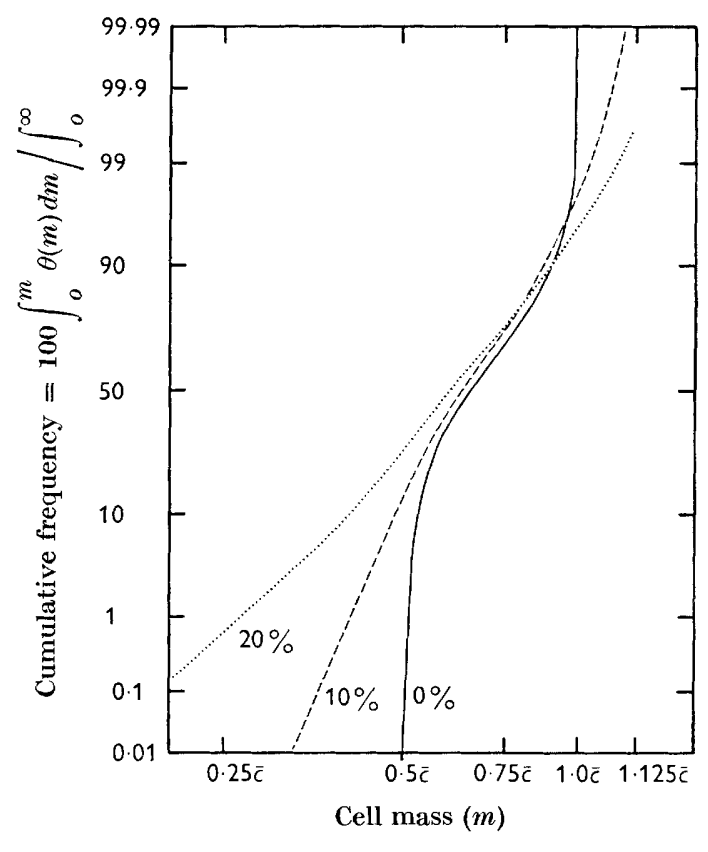

Fig. 2

Fig. 1. Exponential mass increase model. The distribution of cell sizes for a culture in balanced growth where the mass of each cell increases exponentially at the same rate. Curves are presented for coefficients of variation of the size attained at cell division of 0,10 , and $20 \%$. The fluctuations are assumed Gaussian.

Fig. 2. As Fig. 1. but log-probability plot.

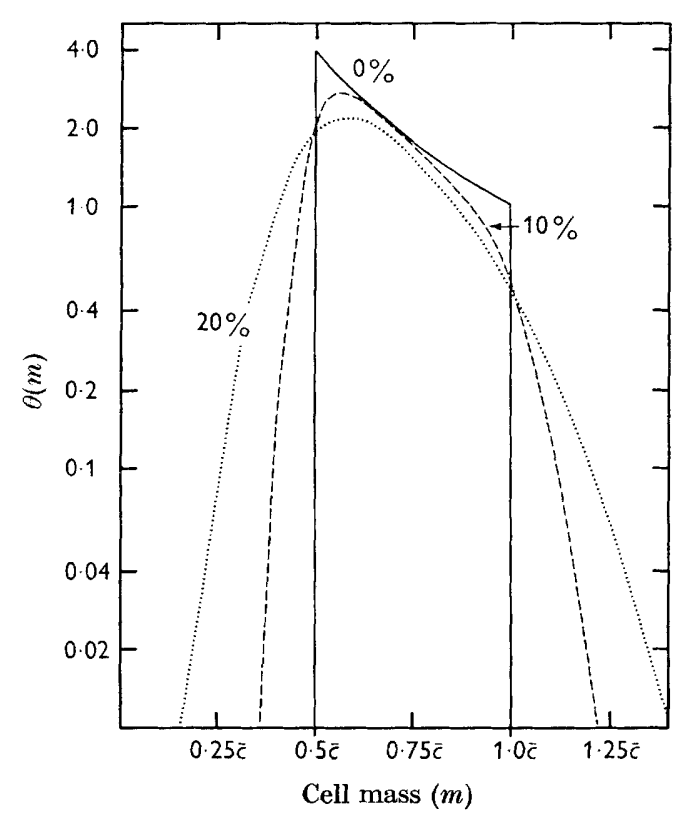

Fig. 3

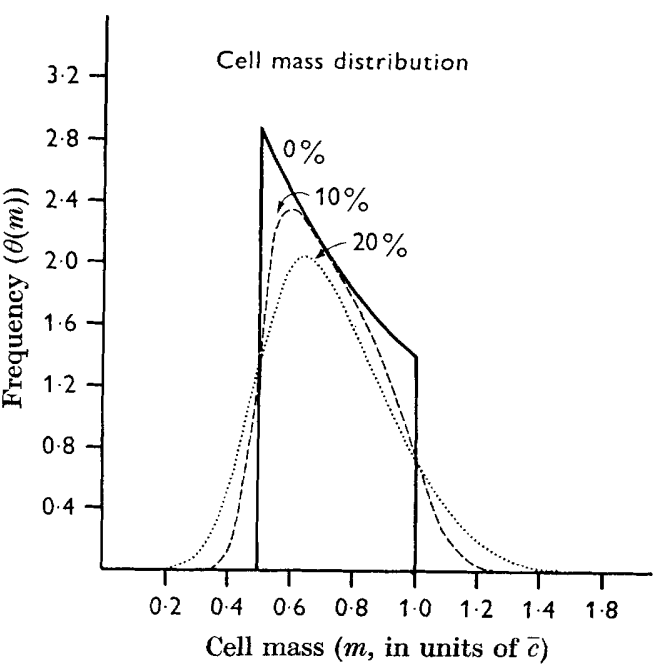

Fig. 4

Fig. 3. As Fig. 1 but in semi-logarithmic representation.

Fig. 4. Linear mass increase model. Other assumptions for the model are given in the legend to Fig. 1 and in the text. 
percentile to the 90 percentile for a $10 \%$ c.v. In Fig. 3 the frequency distribution is presented on a semilogarithmic plot. It is presented in this way since this is a convenient representation and because certain of the instruments print out the data this way. Plots of this kind are convenient for estimating the modal size.

Alternatively, it might be assumed that bacterial protoplasm increases at a constant rate, i.e. growth is linear throughout the cell division cycle. A model, although not a likely one, is that only a fully intact genome may serve as template for messenger, and that the level of messenger limits protoplasm synthesis. There are other models that would also predict linear growth. In any case, this is a reasonably extreme hypothesis from the biochemical point of view. On this hypothesis the rate per unit amount of protoplasm doubles when the cell divides into two entities in order that each one of them continues at the same old rate. If it is further assumed that each cell grows from a mass of exactly $1 / 2 \bar{c}$ to exactly $\bar{c}$ and then divides, the following distribution is obtained:

and

$$
\left.\begin{array}{l}
\theta(m)=\frac{8 \ln 2}{\bar{c}} \exp \left(-\frac{2 m \ln 2}{\bar{c}}\right) ;\left(\frac{1}{2} \bar{c} \leqslant m \leqslant \bar{c}\right) \\
\theta(m)=0 ; \quad\left(m<\frac{1}{2} \bar{c}, m>\bar{c}\right) .
\end{array}\right\}
$$

We do not believe this distribution has been presented in the literature before, but its derivation from the well-known age distribution is quite simple and will be omitted. The mean of this distribution was calculated by Cook \& James (1964). The distribution is shown in Fig. 4.

The mass distribution for the linear growth case when there is a fluctuation in the size at division is also shown in Fig. 4. The formula can be derived in a manner similar to that used by Powell (1964) and is

$$
\theta(m)=C 2 \ln 2 \exp (-2(\ln 2) m / \bar{c}) \int_{0}^{m} \frac{\exp (2(\ln 2) m / \bar{c})}{m}(g(2 m)-g(m)) d m,
$$

where $C$ is still the harmonic mean of the $g(m)$ distribution. Equation (4) becomes equation (3) as the coefficient of variation of the $g(m)$ distribution decreases.

\section{The Collins-Richmond treatment}

Another entirely different approach to this problem was introduced by Collins \& Richmond in 1962. These workers derived a relationship between the rate of protoplasm synthesis of cells of a given size in terms of three distributions: the distribution of cell sizes in the balanced growing population, $\lambda\left(l_{x}\right)$; the distribution of cell sizes as the cells divide, $\varnothing(l)$; and the distribution of cell sizes of those cells that are just formed, $\psi(\mathrm{l})$. Their equation, in their symbolism, is

$$
V_{x}=k\left\{2 \int_{o}^{l_{x}} \psi(e) d l-\int_{o}^{l_{x}} ø(e) d l-\int_{o}^{l_{x}} \lambda(e) d l\right\} / \lambda\left(l_{x}\right) .
$$

Here, $k$ is the growth rate constant.

In the derivation of the size distributions given in equations (1)-(4), it has been assumed that cell division divides the cell precisely into equal halves; and so the latter two distributions, $\psi$ and $ø$ of Collins \& Richmond, are of precisely the same shape. Equation (2) above can be, and has been, suitably modified when this is not true. Since we have been able to show that division in Escherichia coli is quite 
precise (Koch, 1966), only the simpler distributions based on this presumption are given here and are used in the calculations given below. With this simplification, we can write the Collins \& Richmond equation, in terms of the quantities defined in the present paper, approximately as follows:

$$
\theta(m) V_{m}=\mathbf{2} k \int_{o}^{m} \frac{g(\mathbf{2} m) d m}{m}-k \int_{o}^{m} \frac{g(m) d m}{m}-k \int_{o}^{m} \theta(m) d m .
$$

This expression is rigorously correct (see note on p. 416) only if the $g(m)$ distribution is very narrow. We will give no computations based on (6), but present it for comparison purposes.

Using this approach, Collins \& Richmond calculated that the rate of protoplasm synthesis in Bacillus cereus is nearly proportional to the total amount of protoplasm in the individual cell throughout the range of the average cell division cycle, independent of the assumption about the width of the $g(m)$ distribution. Thus, they interpreted their results as being in closer, but not in precise, agreement with the postulate leading to equations (1) and (2) and differing from that leading to equations (3) or (4).

Marr \& Harvey (1965) used a modified Coulter counter for the evaluation of the growth law from the Collins-Richmond principle. While they found deviations at extreme sizes, they also concluded that growth is nearly exponential for cells in the normal range of size. While these are highly reasonable conclusions and ones that fit our preconceived notions, it is one of the purposes of the present note to point out that their conclusions require that the cell size distribution be very accurately and precisely shown.

Table 1. Comparison of exponential and linear growth models for population size distribution

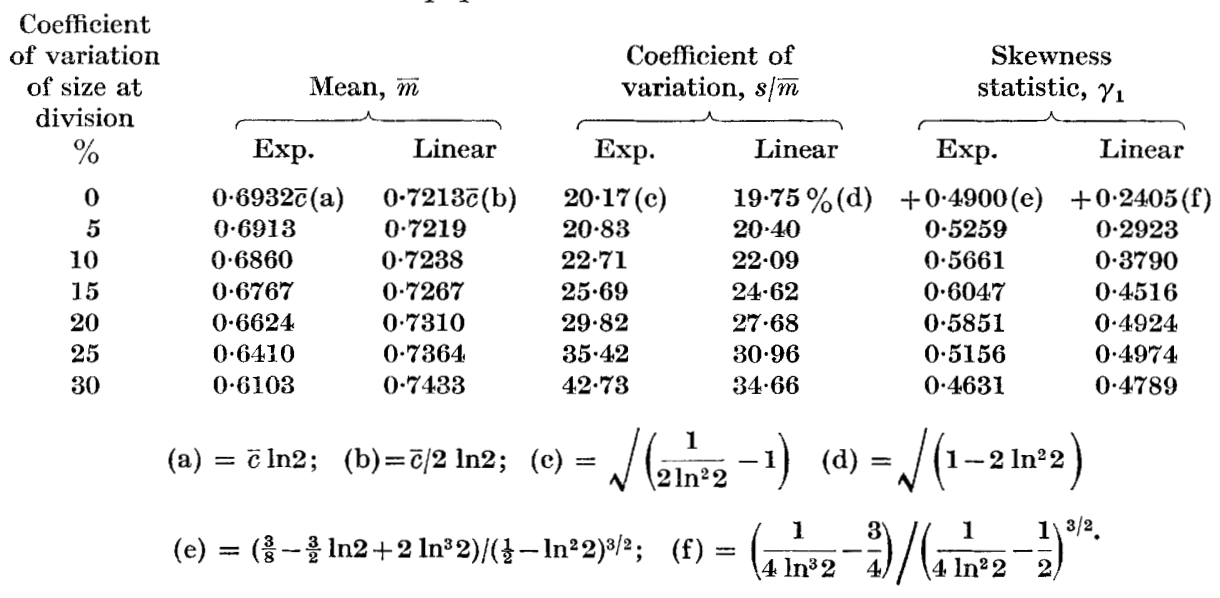

Statistical parameters of the theoretical distributions

Numerical calculations based on equations (1)-(4) are presented in Table 1. These computations were done on the 709 machine at the University of Florida Computing Center. We have assumed that the distribution function of the sizes at division is Gaussian for these calculations and in those on which Figs. 1-4 are based. 
This is an entirely arbitrary presumption. We have also made a judgement as to which of several related yet distinct distributions (see note on p. 416) to take as the normal one (see Powell, 1964, for an exposition). Our basis for the choice is clear and reasonable: as long as we restrict our consideration to relatively small values of c.v. for the size at division, the population size will be relatively insensitive to the shape of the $g(m)$ distribution because of the way in which $g(m)$ enters the calculation. Moreover, the conclusions to be drawn from these computations will be directed solely to the changes in the population size distribution as the type of growth and the dispersion of the distribution of sizes at division are altered.

From the statistical parameters of theoretical distributions given in Table 1, it is seen that it is impossible to deduce anything at all about the growth law by simply measuring the c.v. of the size distribution of cultures in balanced growth. For the case where there is no fluctuation in cell size at division, the c.v. is nearly $20 \%$ for either type of growth. Even if the cell size distribution were very different, the c.v. would not change very much. For example, if the distribution were rectangular from $0 \cdot 5 \bar{c}$ to $\bar{c}$, the c.v. would be $\mathbf{1 9} \cdot 4 \%$. Thus, the dispersion of the cell size distribution is largely set by the fact that a cell divides to yield cells that are half as big. The c.v. is significantly different from $20 \%$ only if there are widely dramatic variations in the rate of protoplasm synthesis during division cycle. Such fluctuations are contrary to the limited experimental evidence available (Schaechter et al. 1962; Hoffman \& Franks, 1965; Cummings, 1965).

Evidently, for its conclusions about the law of protoplasmic synthesis, the CollinsRichmond treatment depends on moments of the distribution higher than the second. Thus, there is a small but significant difference in the third moment about the mean between the two distributions when the size of cells at cell division is precisely determined, but the difference decreases as the fluctuation in the size at cell division is increased.

As fluctuation in the critical size of division is introduced, the measure of skewness, $\gamma_{1}$, at first increases and then decreases. It increases because the third moment about the mean increases as the distribution spreads out at both extremes. The decrease is due to the increase in the standard deviation of the distribution. Since the skewness statistic, $\gamma_{1}$, is defined as the third moment about the mean divided by the cube of the standard deviation, a moderate increase in the standard deviation leads to a decrease in $\gamma_{1}$.

In the absence of experimental bias or error, the standard error of the $\gamma_{1}$ statistic is nearly $\sqrt{ }(6 / n)$. Thus, 600 cells would be enough to establish a distinction if there were $0 \%$ fluctuation in cell size at division, and 60,000 would be barely enough with $30 \%$ coefficient of variation of division size.

We now return to the most accurately determinable parameter of the distribution of population sizes, namely, its coefficient of variation. It is seen from Table 1 that for either exponential or linear growth, $10 \%$ of fluctuation at division adds only $3 \%$ to the c.v. of the basic distribution, whereas a $20 \%$ fluctuation in size at division adds 8 to $10 \%$ to the basic fluctuation. This leads to the important conclusion that the coefficient of variation of an observed distribution of sizes in balanced growth is a function of the fluctuation of size at division but is almost independent of the kind of growth law. 
A CRITERION FOR THE POPULATION SIZE DISTRIBUTION

As far as the author is aware, fluctuation in division size of enteric bacteria at division rarely exceeds $15 \%$ under conditions with normal genotypes in true balanced growth (Schaechter et al. 1962), so the coefficient of variation of size distribution should not exceed about $26 \%$. If a broader cell size distribution than $30 \%$ is found, either experimental artifacts have contributed to broadening the distribution or there are abnormally large fluctuations in the size of the bacteria at division. The resolution between these two possibilities would be necessary before a successful attempt to elucidate the growth law would be possible.

To document this further, we have calculated the c.v. of bacterial size distribution in balanced growth from published and unpublished data available to us (Table 2). Sets of data were used in which various organisms and various experimental techniques were used. All, however, involve visual examination of the bacteria, so that artifacts resulting from the adherence of sister bacteria are presumably not present. In two cases the lengths only were measured and the diameters of the rod-shaped bacteria have been presumed constant. In the other case, both lengths and widths were measured from electron micrographs and volumes computed. We have calculated the coefficients of variation from the workers' published histograms. It is evident that all of these microscopically observed cases fit together with the hypothesis that the critical size at division has a coefficient of variation of not more than $15 \%$, independent of any detailed hypothesis of the nature of the growth law between the division events.

Table 2. Coefficient of variation of experimental distributions of bacterial sizes during balanced growth

$\begin{array}{lccc} & \begin{array}{c}\text { Type of } \\ \text { Keasurement }\end{array} & \text { Number } & \begin{array}{c}\text { Coefficient } \\ \text { of variation } \\ (\%)\end{array} \\ \begin{array}{l}\text { Kubitschek \& Bendigkeit (1955) } \\ \text { Escherichia coli } \text { B }\end{array} & \text { Volumes } & 390 & 25 \cdot 53 \\ \begin{array}{l}\text { Schaechter (unpublished 1960) } \\ \quad \text { E. coli } \text { B } / \mathbf{r}\end{array} & \text { Lengths } & 206 & 21 \cdot 03 \\ \begin{array}{c}\text { Salmonella typhimurium } \\ \text { Collins \& Richmond (1962) }\end{array} & \text { Lengths } & 151 & 20 \cdot 58 \\ \text { Bacillus cereus } & \text { Lengths } & 1932 & 22.63\end{array}$

\section{THE NEED FOR CAUTION IN INTERPRETING EXPERIMENTAL SIZE DISTRIBUTIONS}

The application of the Coulter principle opens new vistas to the study of general physiology, since it can, in principle, accurately define both the control of the division process and the kinetics of protoplasm synthesis at the single cell level. In addition, it can function with particles of the size of bacteria, which it may be hoped will follow simpler laws than may apply to bigger or more differentiated organisms. It can only do this if the instrument allows an accurate estimate of size as well as yielding an accurate estimate of the number of organisms. The latter is achieved simply because a statistically large number of bacteria can be quickly enumerated. The physics of the resistance changes are by no means simple (see, for 
example, Gregg \& Steidley, 1965). They show that there can be as much as a $50 \%$ difference in the resistance change of particles of the same volume in going from a spherical shape to a long rod. Evidently, the growth of enteric bacteria by elongation of the rod at constant diameter could lead to a distortion of the size distribution. Other difficulties could be mentioned. The most serious one is that alluded to above, i.e. the possibility that two daughters which have just arisen from the division of a parent remain attached for a variable length of time and thus artificially broaden the distribution. Also very serious is the fact, as pointed out by Kubitschek (1964), that the transit time varies from a minimum for those particles which go down the aperture axis to very slow passage in the laminar flow near the edges of the orifices. This can mean that with certain electronic circuits the size of those particles which go near the axis will be underestimated relative to the others. Other difficulties may emerge as further work is carried out.

On the other hand, microscopic observations are not without serious drawbacks. The measurements are very laborious, and it is very difficult to accumulate enough data on a single population to achieve a highly accurate size distribution. Necessarily, only two dimensions can be measured and the third must be inferred. In some cases, preparation artifacts due to drying and staining will be introduced. Still, careful measurements with the optical methods can serve as primary standards to make sure that the automatic devices are giving a reliable picture.

The proposal outlined above should serve as a check of the overall instrumentation, if only as a first and crude test. We simply propose that the coefficient of variation of the size distribution for enteric bacteria in balanced growth under conditions in which the organisms do not 'snake' should be measured on every apparatus and under the range of conditions to be used. The results should be a value consonant with those indicated in Table 1 . Then, and only then, should the effects of drugs or physiological conditions be studied or an analysis based on the precise shape of the distribution be undertaken. This criterion has been met, at least for some conditions, in the apparatus of A. G. Marr (personal communication), and it would be reasonable to expect that such coefficients of variation be control data to be included with reports about cell size distributions recorded with the Coulter principle.

These considerations arose as the result of action and reaction to $\mathbf{H}$. Kubitschek, A. G. Marr, and M. Schaechter; and I wish to express my gratitude for their helping me to work on a problem that is theirs and not mine. Experimental work in the author's laboratory is supported by U.S. Public Health Service grant CA-07404 and National Science Foundation grant GB-4.538.

\section{NOTE}

I have taken $g(m)$ to be the distribution function for the sizes at division. The $\varnothing$ and $\psi$ function in the Collins \& Richmond treatment are also distribution functions for the sizes at division, but relate to a different population of cells. $(g(m)) / m$ is approximately $\varnothing(m)$, but $(g(m)) / m$ cannot be a true distribution function since its integral cannot be unity at the same time that the integral of $g(m)$ is unity. Evidently, no error is involved if $g(m)$ is a very narrow distribution. I wish to thank 
Dr A. G. Marr very much for pointing this out and for sending me his unpublished calculations based entirely on the Collins-Richmond equation. His calculations do not involve the approximations involved in equations (2) and (4). Comparing his calculated values with ours, it is seen that appreciable error results only when the c.v. of $g(m)$ is greater than $20 \%$. Even then the error is mainly in the mean value of the distribution and to a much lesser degree in c.v. and $\gamma_{1}$ values.

\section{REFERENCES}

Collins, J. F. \& Richmond, M. H. (1962). Rate of growth of Bacillus cereus between division. J. gen. Microbiol. 28, 15.

Соок, J. R. \& J JMes, T. W. (1964). Age distribution of cells in logarithmically growing cell populations. In Synchrony in Cell Division and Groveth, p. 405. Ed. by E. Zeuthen. New York: Interscience.

Cummings, D. J. (1965). Macromolecular synthesis during synchronous growth of Escherichia coli B/r. Biochim. biophys. Acta 95, 341.

GregG, E. C. \& Steidley, K. D. (1965). Electrical counting and sizing of mammalian cells in suspension. Biophys. $J .5,393$.

Hofman, H. \& Franks, M. E. (1965). Time-lapse photomicrography of cell growth and division in Escherichia coli. J. Bact. 89, 212.

Koch, A. L. (1966). On evidence supporting a deterministic process of bacterial growth. J. gen. Microbiol. 43, 1.

Koch, A. L. \& Schaechter, M. (1962). A model for statistics of the cell division process. J. gen. Microbiol. 29, 435 .

Kubitscheк, H. E. (1964). Apertures for Coulter counters. Rev. scient. Instrum. 35, 1598.

Kubitscheк, H. E. \& Bendigkeit, H. E. (1955). Some studies on the growth and size of bacteria. Argonne National Laboratories, 5426, 99.

McLean, F. I. \& Munson, R. J. (1961). Some environmental factors affecting the length of Escherichia coli organisms in continuous cultures. J. gen. Microbiol. 25, 17.

Marr, A. G. \& Harvey, R. J. (1965). Kinetics of growth of individual cells. Bact. Proc. p. 38.

Powelt, E. O. (1964). A note on Koch and Schaechter's hypothesis about growth and fission of bacteria. J. gen. Microbiol. 37, 231.

Schaechter, M., Williamson, J. P., Hoon, J. R., Jr. \& Koch, A. L. (1962). Growth, cell and nuclear divisions in some bacteria. J. gen. Microbiol. 29, 421. 